\title{
Extraction of the attosecond time delay in atomic photoionization using the soft-photon approximation
}

\author{
I. A. Ivanov* and A. S. Kheifets \\ Research School of Physical Sciences, Australian National University, Canberra, Australian Capital Territory 0200, Australia
}

(Received 25 April 2013; revised manuscript received 29 May 2013; published 20 June 2013)

\begin{abstract}
We use the soft-photon approximation to extract the Wigner time delay from atomic two-color photoionization experiments. Unlike the strong-field approximation, the present method does not require introduction of the Coulomb-laser coupling corrections and enables one to extract the Wigner time delay directly from attosecond time delay measurements.
\end{abstract}

DOI: 10.1103/PhysRevA.87.063419

PACS number(s): 32.80.Fb, 32.30.Rj, 32.70.-n, 31.15.ve

\section{INTRODUCTION}

The concept of time delay was developed in formal scattering theory by Wigner [1] and his contemporaries (see Ref. [2] for a comprehensive review). It is a quantity related to the phase of the complex scattering amplitude which provides insight into development of the scattering process in time. In recent years, this idea has made a dramatic comeback when it was realized that the time delay can be measured experimentally in photoionization processes. This has led to many interesting and not yet fully understood results such as observation of a considerable time delay between photoelectrons emitted from the $2 s$ and $2 p$ subshells in neon [3] and the experimental determination of the tunneling time in an ionization event [4].

The timing information in photoionization process is extracted experimentally by applying an ionizing XUV pulse (the pump pulse) and an infrared (IR) probe pulse. In the attosecond streaking experiments, the time delay between the pump and probe pulses is mapped onto the kinetic energy of the photoelectron in the form of a spectrogram. In such experiments, duration of the probe pulse may be several optical cycles of the IR field [3]. Alternatively, one may use the socalled reconstruction of attosecond bursts by ionization of twophoton transitions (RABITT) technique [5], which employs a monochromatic IR probe. In this technique, the pump-probe delay is mapped onto the phase of the sideband oscillations caused by interference of alternative two-photon ionization processes. A detailed description of these techniques can be found in Ref. [6].

To extract the Wigner time delay related to the XUV photoionization, one has to take into account the effect of the probe IR field on the system under investigation. In the RABITT experiments with monochromatic probes, the IR field is typically weak, which allows the perturbation theory treatment $[7,8]$. In the attosecond streaking approach, where the IR probe intensity is typically in the range of $10^{11}$ to $10^{12} \mathrm{~W} / \mathrm{cm}^{2}$, the nonperturbative treatment is called for. In the first interpretation of the attosecond streaking experiment [9], the well-known classical equation was invoked:

$$
\boldsymbol{p}_{f}(t)=\boldsymbol{p}_{0}-\boldsymbol{A}^{\mathrm{IR}}(t)
$$

relating the unperturbed asymptotic momentum of the photoelectron $\boldsymbol{p}_{0}$ and the final momentum $\boldsymbol{p}_{f}(t)$ for emission

*igor.ivanov@anu.edu.au at time $t$ in the presence of an IR field $\boldsymbol{A}^{\mathrm{IR}}$. This implies that the interaction of the photoelectron with the ionic core is neglected. To account for the corrections due to this interaction (the so-called Coulomb-laser coupling), a further refinement of this model has been developed [10-12]. This refinement results in renormalizing the vector potential in Eq. (1) and shifting its argument by adding the the so-called Coulomb-laser coupling correction in the argument of the function $\boldsymbol{A}^{\mathrm{IR}}(t)$. In Ref. [13] the authors were able to derive a similar formula using an analytical approach based on the eikonal approximation. Yet another approach, based on the direct numerical evaluation of the quantity defining the time delay (which is essentially the additional time the particle would spend in a region of space as compared to the free particle) has been proposed in Ref. [14].

Below we present an alternative procedure of extraction of the time delay from the experimentally observable photoionization cross sections. This procedure introduces an accurate description of the IR field influence from the outset and no further corrections are needed.

\section{THEORY AND COMPUTATIONAL DETAILS}

The procedure is based on the so-called soft-photon approximation [15]. Under condition of the IR photon frequency being small in comparison with the photoelectron energy, this approximation has been shown to reproduce quite accurately the angle-integrated cross sections of the process of two-color ionization by the XUV and IR fields [16]. To extract timing information, one has to know the phase or, rather, the energy derivative of the phase of the amplitude of the ionization process. It is unclear whether the soft-photon approximation can cope with this problem. Below, we address this question.

We consider a typical configuration of the XUV and IR fields used in the attosecond streaking experiments. The time dependence of the electric field of the IR pulse is

$$
\mathcal{E}^{\mathrm{IR}}(t)=\mathcal{E}_{0}^{\mathrm{IR}} \sin \Omega t
$$

with the base frequency $\Omega=0.057$ a.u. (photon energy of $1.55 \mathrm{eV})$. The IR field is present on the interval of time $\left(0, T_{1}\right)$, where $T_{1}=2 \pi / \Omega=2.7 \mathrm{fs}$ is an optical cycle corresponding to the IR frequency $\Omega$.

The XUV pulse is present on the time interval $\Delta-4 T, \Delta+$ $4 T$, where $T=2 \pi / \omega$ is an optical cycle of the XUV pulse. Parameter $\Delta$, therefore, characterizes the relative shift between beginning of the IR pulse and arrival of the center of the XUV 
pulse. On this interval the XUV field time dependence is

$$
\mathcal{E}^{\mathrm{XUV}}(t)=\mathcal{E}_{0}^{\mathrm{XUV}} f\left(t^{\prime}\right) \cos \omega t^{\prime},
$$

where $t^{\prime}=t-\Delta$, and we use a cosine squared envelope function $f\left(t^{\prime}\right)=\cos ^{2}\left(\omega t^{\prime} / 16\right)$. The XUV field strength is $\mathcal{E}_{0}^{\mathrm{XUV}}=0.01$ a.u. (intensity of $3.5 \times 10^{12} \mathrm{~W} / \mathrm{cm}^{2}$ ). Both pulses are assumed to be linearly polarized along the $z$ axis. As a target system, we consider the $\mathrm{Ne}$ atom described by a localized model potential [17] within the single active electron (SAE) approximation.

The amplitude of the photoionization process can be defined as

$$
f(\boldsymbol{k})=\lim _{\substack{t \rightarrow \infty \\ \tau \rightarrow-\infty}} e^{i\left[E(k) t-E_{0} \tau\right]}\left\langle\Psi_{\boldsymbol{k}}^{-} \mid \hat{U}(t, \tau) \phi\right\rangle,
$$

where $\Psi_{k}^{-}$is the (ingoing) scattering wave function describing the photoelectron with the kinetic energy $E(k), \hat{U}(t,-\infty)$ is the evolution operator propagating the system in the presence of the IR and XUV fields, $\phi$ is the initial atomic state, and $E_{0}$ is its energy. For a relatively weak XUV field strength, the photoionization amplitude in the presence of the XUV pulse alone is given by the well-known perturbative formula:

$$
f^{\mathrm{XUV}}(\boldsymbol{k})=-i \int_{-\infty}^{\infty}\left\langle\Psi_{\boldsymbol{k}}^{-}\left|\hat{H}_{\mathrm{int}}^{\mathrm{XUV}}(t)\right| \Psi_{0}\right\rangle e^{i\left(E(k)-E_{0}\right) t} d t .
$$

Expression for the evolution operator applicable for a weak XUV field can be obtained from the Dyson equation:

$$
\hat{U}(t, \tau)=\hat{U}_{0}(t, \tau)-i \int_{-\infty}^{t} \hat{U}_{0}\left(t, \tau^{\prime}\right) H_{\mathrm{int}}^{\mathrm{XUV}}\left(\tau^{\prime}\right) \hat{U}_{0}\left(\tau^{\prime}, \tau\right) d \tau^{\prime},
$$

where $\hat{U}_{0}(t, \tau)$ is the evolution operator for the atom in presence of the IR field only. In the following, we adopt the CoulombVolkov approximation (CVA) $[18,19]$. In this approximation, the action of the evolution operator $U_{0}(\tau, t)$ on the scattering state $\Psi_{k}^{-}$of the atom is expressed as

$$
\hat{U}_{0}(\tau, t) \Psi_{\boldsymbol{k}}^{-}=\Psi_{\boldsymbol{k}}^{-} \exp \left\{-\frac{i}{2} \int_{t}^{\tau}\left[\boldsymbol{k}+\boldsymbol{A}^{\mathrm{IR}}\left(\tau^{\prime}\right)\right]^{2} d \tau^{\prime}\right\},
$$

where $A^{\mathrm{IR}}(t)=-\int_{0}^{t} \mathcal{E}^{\mathrm{IR}}(\tau) d \tau$ is the vector potential of the IR field. We also make an assumption that the IR field perturbs the initial (ground) state only slightly. So, we can write $\hat{U}_{0}\left(\tau^{\prime}, \tau\right) \phi=e^{-i E_{0}\left(\tau^{\prime}-\tau\right)} \phi$.

We consider below emission of the photoelectron in the $z$ direction, which is parallel to the polarization vectors of both the IR and XUV fields. By substituting Eq. (6) into (5), by using the CVA, expanding exponential introduced by the CVA as a Fourier series, and utilizing the perturbative equation Eq. (5) for the photoionization amplitude in the presence of the XUV field only, we obtain the following relation between the amplitude $f\left(k_{z}, \Delta\right)$ of the two-color ionization and the amplitude $f^{\mathrm{XUV}}\left(k_{z}, \Delta\right)$ of the photoionization process driven by the XUV field alone:

$$
f\left(k_{z}, \Delta\right)=e^{i[y-E(k)] T_{1}} \sum_{m=-\infty}^{\infty} J_{m}\left(\frac{y}{\Omega}\right) f^{\mathrm{XUV}}\left(k_{z}^{(m)}, \Delta\right),
$$

where $y=\mathcal{E}_{\mathrm{IR}}^{0} k_{z} / \Omega, k_{z}^{(m)}=\sqrt{k^{2}-2 y+2 m \Omega}$, and $J_{m}$ is a Bessel function. Terms with different $m$ in Eq. (8) describe processes with participation of $m$ IR photons.
By using Eq. (8) for various delays $\Delta$ between the IR and XUV fields, we can obtain a set of relations between $f\left(k_{z}, \Delta\right)$ and $f^{\mathrm{XUV}}\left(k_{z}, \Delta\right)$. The perturbative expression (5) allows us to express $f^{\mathrm{XUV}}\left(k_{z}, \Delta\right)$ in terms of the "reference" amplitude $f^{\mathrm{XUV}}\left(k_{z}, 0\right)$ as $f^{\mathrm{XUV}}\left(k_{z}, \Delta\right)=e^{i\left(E(k)-E_{0}\right) \Delta} f^{\mathrm{XUV}}\left(k_{z}, 0\right)$. We can thus relate the two-color amplitudes $f\left(k_{z}, \Delta\right)$ and the reference amplitude $f^{\mathrm{XUV}}\left(k_{z}, 0\right)$ for different values of $\Delta$.

Our goal is to determine the phase, or rather the phase derivative, of the reference amplitude with respect to the electron momentum, since the quantity of interest for us, the time delay $\tau_{0}$, can be expressed as [20]

$$
\tau_{0}=\frac{1}{k_{z}} \operatorname{Im}\left(\frac{\partial f^{\mathrm{XUV}}\left(k_{z}, 0\right)}{\partial \boldsymbol{k}_{z}}\right) .
$$

Here the derivative is to be taken at the point $k_{z}$ satisfying the energy conservation $E_{0}+\omega=k_{z}^{2} / 2, E_{0}$ being the energy of the initial atomic state. By using this equation and Eq. (8), it is not difficult to devise a procedure to obtain information about the phase of the reference amplitude for the process of photoionization by the XUV field from the experimentally measurable cross sections of the photoionization process in the presence of both the XUV and IR fields. Before describing implementation of such a procedure, we have to ascertain first that Eq. (8) is accurate enough.

\section{NUMERICAL RESULTS}

We solve the time-dependent Schrödinger equation (TDSE) for the $\mathrm{Ne}$ atom described by means of a localized one-electron potential [17] in the presence of the XUV and IR fields given by Eqs. (2) and (3). We employ the procedure to solve numerically a three-dimensional (3D) TDSE, which is described in detail in Refs. [20,21]. By projecting the solution of the TDSE on the scattering state $\Psi_{k}^{-}$of the Ne atom, as prescribed by Eq. (4), we obtain the photoionization amplitude $f(\boldsymbol{k})$ in the presence of both the XUV and IR fields. A separate calculation of atomic evolution in the presence of the XUV pulse alone described by Eq. (3) with $\Delta=0$ gives us a "reference" amplitude $f^{\mathrm{XUV}}\left(k_{z}, 0\right)$. By using the relation connecting $f^{\mathrm{XUV}}\left(k_{z}, \Delta\right)$ and $f^{\mathrm{XUV}}\left(k_{z}, 0\right)$ and Eq. (8), we can compute values of $f\left(k_{z}, \Delta\right)$, which is the amplitude of the two-color ionization for different values of the delay $\Delta$ between the XUV and IR pulses, and compare them with the $a b$ initio values of $f\left(k_{z}, \Delta\right)$ provided by the TDSE calculation. Such a comparison is shown in Figs. 1-5. The data were obtained retaining the terms with $|m| \leqslant 3$ in Eq. (8) for $\mathcal{E}_{\mathrm{IR}}^{0}=0.001$ a.u. and $|m| \leqslant 5$ for $\mathcal{E}_{\mathrm{IR}}^{0}=0.004$ a.u. The number of the terms which are to be retained in Eq. (8) depends on the strength of the IR field and can be easily determined for each particular value of $\mathcal{E}_{\mathrm{IR}}^{0}$ by checking that convergence with respect to $m$ is achieved.

The data displayed in these figures show that Eq. (8) allows us to compute values of the two-color ionization amplitude $f\left(k_{z}, \Delta\right)$ with a reasonable accuracy for ionization from $2 s$ and $2 p$ states of Ne provided we know the reference amplitude $f^{\mathrm{XUV}}\left(k_{z}, 0\right)$ as a function of the momentum. Results, as can be surmised from the figures, are slightly more accurate for smaller IR field intensities.

We now try to solve an inverse problem of the reconstruction of the amplitude $f^{\mathrm{XUV}}\left(k_{z}, 0\right)$ provided that absolute values 

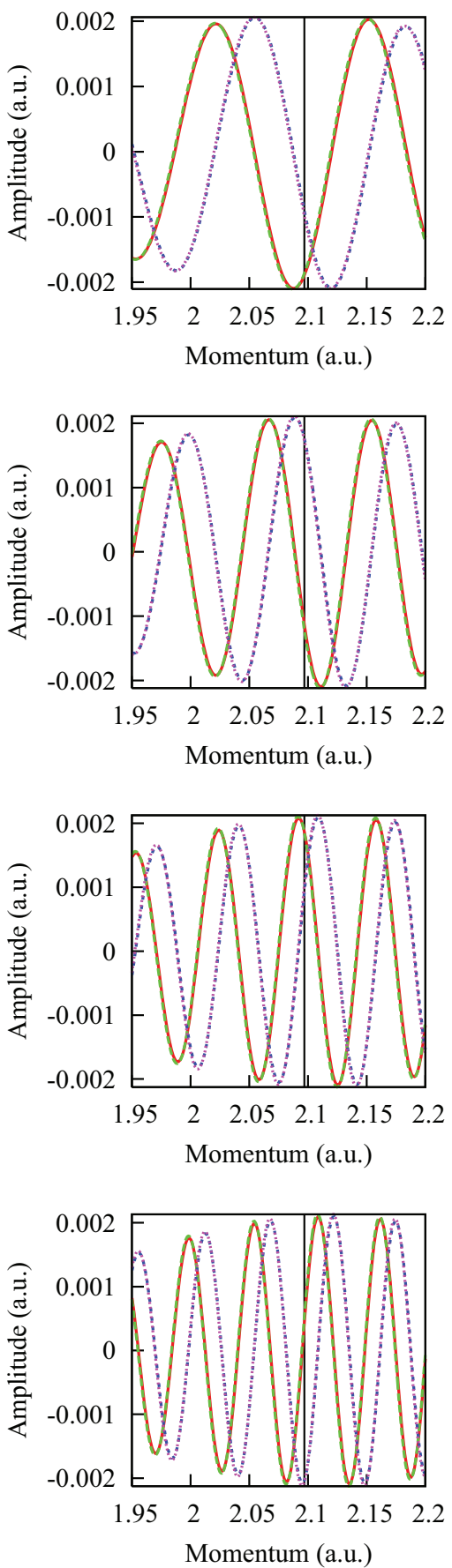

FIG. 1. (Color online) Ionization from the $2 s$ and state of a $\mathrm{Ne}$ atom. $\operatorname{Re} f\left(k_{z}, \Delta\right)$ computed using Eq. (8), solid (red online) line, and TDSE calculation, dashed (green online) line. $\operatorname{Im} f\left(k_{z}, \Delta\right)$ given by Eq. (8), short dashed (blue online) line, and TDSE, dotted (magenta online) line. XUV photon energy $\omega=106 \mathrm{eV} ; \mathcal{E}_{\mathrm{IR}}^{0}=0.001$ a.u. Delays $\Delta$ are (top to bottom) $0.2 T, 0.3 T, 0.4 T$, and $0.5 T$, where $T$ is an optical cycle of the XUV pulse. Vertical solid line corresponds to the momentum $k_{z}$ for which $E_{0}+\omega=k_{z}^{2} / 2$.

of the two-color amplitudes $f\left(k_{z}, \Delta\right)$ are known for some selected values of the delays $\Delta$ and momenta $k_{z}$. This can be demonstrated as follows. We choose a trial form for the amplitude $f^{\mathrm{XUV}}\left(k_{z}, 0\right)$ :

$$
f^{\mathrm{XUV}}\left(k_{z}, 0\right)=A e^{-a(E-\epsilon)^{2}+i \tau(E-\epsilon)},
$$
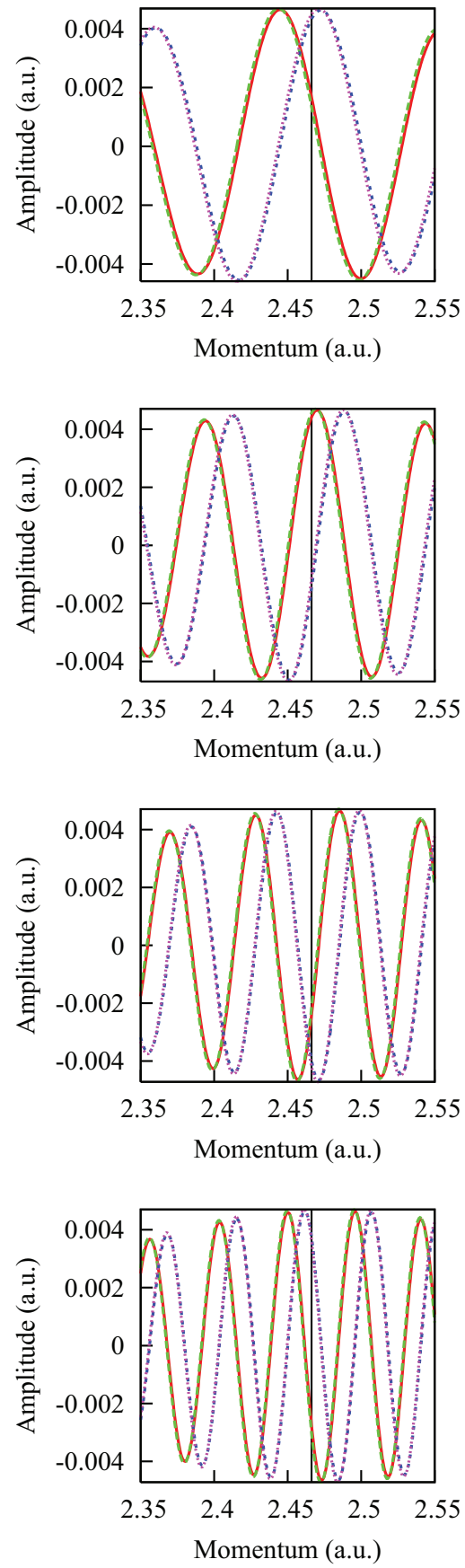

FIG. 2. (Color online) Ionization from the $2 p$ and state of a $\mathrm{Ne}$ atom. $\operatorname{Re} f\left(k_{z}, \Delta\right)$ computed using Eq. (8), solid (red online) line, and TDSE calculation, dashed (green online) line. $\operatorname{Im} f\left(k_{z}, \Delta\right)$ given by Eq. (8), short dashed (blue online) line, and TDSE, dotted (magenta online) line. XUV photon energy $\omega=106 \mathrm{eV} ; \mathcal{E}_{\mathrm{IR}}^{0}=0.001$ a.u. Delays $\Delta$ are (top to bottom) $0.2 T, 0.3 T, 0.4 T$, and $0.5 T$, where $T$ is an optical cycle of the XUV pulse. Vertical solid line corresponds to the momentum $k_{z}$ for which $E_{0}+\omega=k_{z}^{2} / 2$.

where $E=k_{z}^{2} / 2 ; a, \tau$, and $\epsilon$ are fitting parameters; and $A$ is a complex number which does not depend on the energy $E$. Parameter $\epsilon$ has a meaning of the energy at which the cross section of the photoionization by the XUV pulse is peaked. The first guess for the value of this parameter can be obtained from the energy conservation $E_{0}+\omega=\epsilon_{0}$. We could fix the 

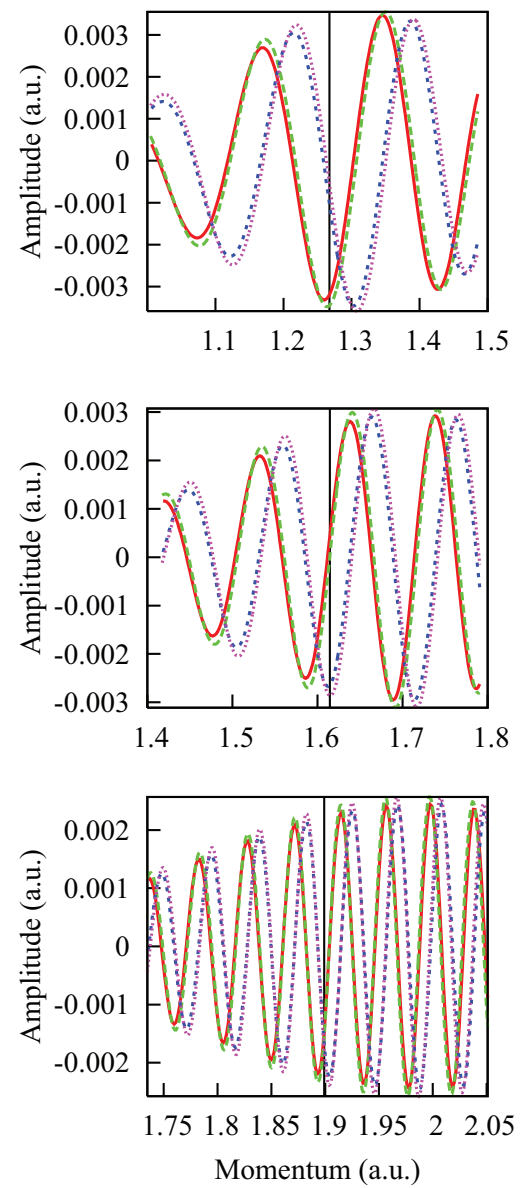

FIG. 3. (Color online) Ionization from the $2 s$ state of a Ne atom. $\operatorname{Re} f\left(k_{z}, \Delta\right)$ computed using Eq. (8), solid (red online) line, and TDSE calculation, dashed (green online) $\operatorname{line} . \operatorname{Im} f\left(k_{z}, \Delta\right)$ given by Eq. (8), short dashed (blue online) line, and TDSE, dotted (magenta online) line. XUV photon energies (top to bottom) are $\omega=68,81.6$, and $95 \mathrm{eV}, \mathcal{E}_{\mathrm{IR}}^{0}=0.004$ a.u. Delays $\Delta$ are (top to bottom) $0.2 T, 0.3 T$, and $0.7 T$, where $T$ is an optical cycle of the XUV pulse. Vertical solid line corresponds to the momentum $k_{z}$ for which $E_{0}+\omega=k_{z}^{2} / 2$.

value of this parameter to $\epsilon_{0}$. However, more accurate results are obtained if we treat it as a fitting parameter. The parameter $\tau$, as can be immediately seen from the Eq. (9), has a meaning of the time delay.

The ansatz (10) does a very good job at reproducing the amplitude $f^{\mathrm{XUV}}\left(k_{z}, 0\right)$, as Fig. 6 testifies. This figure shows comparison of a fit using the functional form (10) to the "exact" amplitude $f^{\mathrm{XUV}}\left(k_{z}, 0\right)$, which we obtain from the TDSE solution for ionization of the $2 s$ subshell of the $\mathrm{Ne}$ atom by the XUV pulse.

By using Eqs. (8) and (10), we can compute the trial amplitude $f^{t}\left(k_{z}, \Delta\right)$ and, consequently, the trial electron spectrum $P^{t}\left(k_{z}, \Delta\right)$ of the two-color ionization of the Ne atom as a function of the momentum for various values of the delay $\Delta$ between the IR and XUV pulses. Using this distribution we can compute the trial expectation values of the electron momentum $\bar{k}_{z}^{t}(\Delta)$ for various $\Delta$,

$$
\bar{k}_{z}^{t}(\Delta)=\int P^{t}\left(k_{z}, \Delta\right) k_{z} d k_{z}
$$
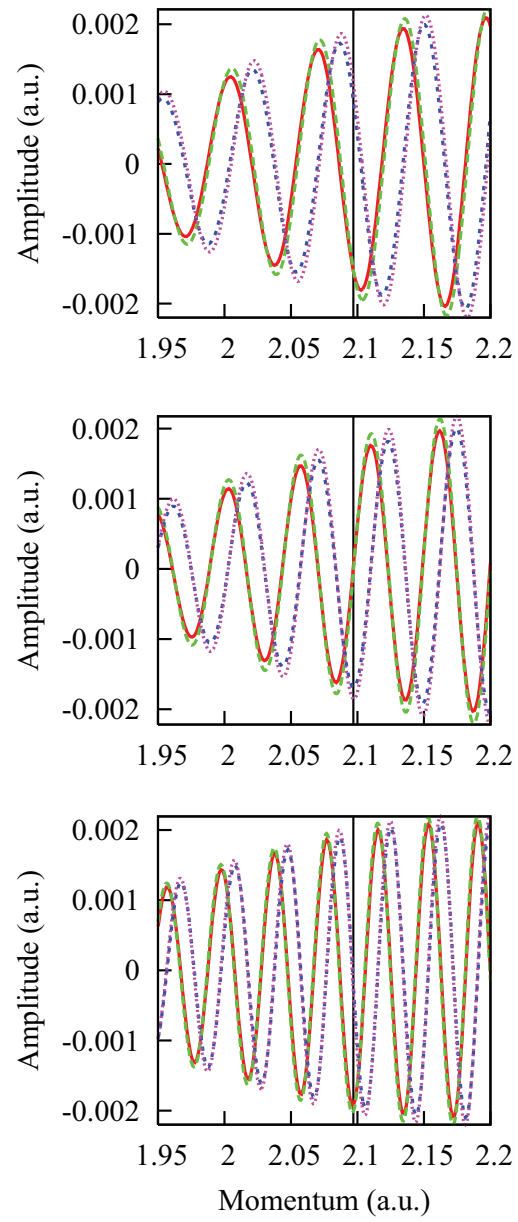

FIG. 4. (Color online) Ionization from the $2 s$ state of $\mathrm{Ne}$ atom. $\operatorname{Re} f\left(k_{z}, \Delta\right)$ computed using Eq. (8), solid (red online) line, and TDSE calculation, dashed (green online) line. $\operatorname{Im} f\left(k_{z}, \Delta\right)$ given by Eq. (8), short dashed (blue online) line, and TDSE, dotted (magenta online) line. XUV photon energy $\omega=106 \mathrm{eV} ; \mathcal{E}_{\mathrm{IR}}^{0}=0.004$ a.u. Delays $\Delta$ (top to bottom) are $\Delta=0.4 T, \Delta=0.5 T, \Delta=0.7 T$, where $T$ is an optical cycle of the XUV pulse. Vertical solid line corresponds to the momentum $k_{z}$ for which $E_{0}+\omega=k_{z}^{2} / 2$.

and compare them with the values $\bar{k}_{z}(\Delta)$ which we obtain from the TDSE calculation (and which can be measured in the experiment).

We can now form a functional:

$$
D(a, \tau, \epsilon)=\sum_{i}\left(\bar{k}_{z}^{t}\left(\Delta_{i}\right)-\bar{k}_{z}\left(\Delta_{i}\right)\right)^{2} .
$$

Sum in Eq. (12) is taken over a set of delays $\Delta$ between the IR and XUV pulses, for which the data are available. Presently, we use the set $\Delta=0.2 T_{1}, 0.3 T_{1}, 0.4 T_{1}, 0.5 T_{1}$, and $0.7 T_{1}$. By minimizing the functional (12) with respect to the parameters $a, \tau$, and $\epsilon$ in Eq. (10), we obtain the time delay $\tau$.

Convergence of this procedure is illustrated in Fig. 7 displaying the function $D^{-1}\left(a, \tau, \epsilon_{0}\right)$ for fixed value of $\epsilon=\epsilon_{0}$, where $\epsilon_{0}=E_{0}+\omega$ is the value given by the energy conservation. This figure shows that $D^{-1}\left(a, \tau, \epsilon_{0}\right)$ has a well-defined pronounced maximum in the space of the parameters $a, \tau$. This property is very useful since it implies that $D^{-1}(a, \tau, \epsilon)$ in Eq. (12) has a deep minimum, which is easy to locate. 

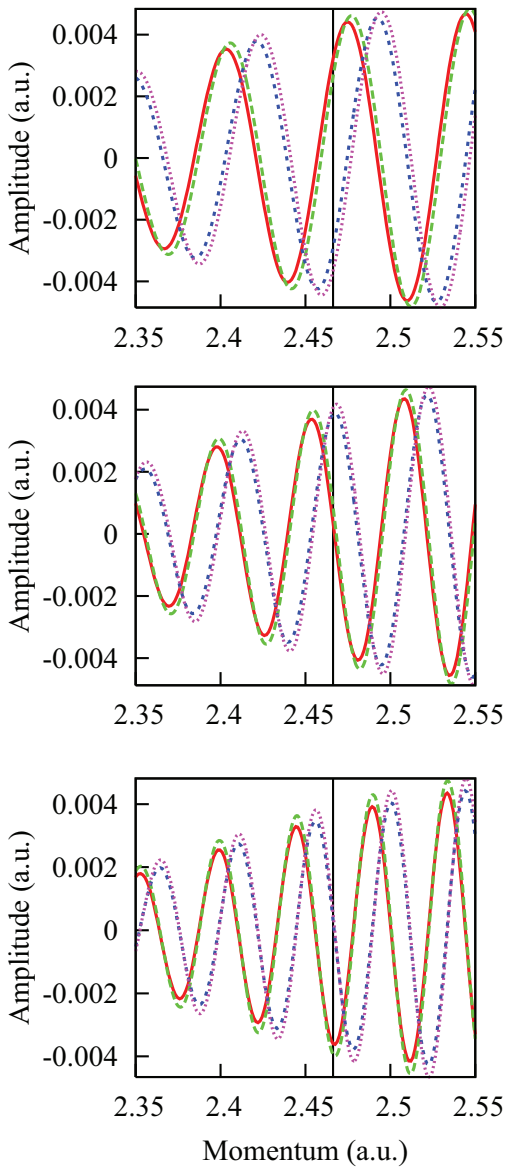

FIG. 5. (Color online) Ionization from the $2 p$ state of $\mathrm{Ne}$ atom. $\operatorname{Re} f\left(k_{z}, \Delta\right)$ computed using Eq. (8), solid (red online) line, and TDSE calculation, dashed (green online) line. $\operatorname{Im} f\left(k_{z}, \Delta\right)$ given by Eq. (8), short dashed (blue online) line, and TDSE, dotted (magenta online) line. XUV photon energy $\omega=106 \mathrm{eV} ; \mathcal{E}_{\mathrm{IR}}^{0}=0.004$ a.u. Delays $\Delta$ (top to bottom) are $\Delta=0.3 T, \Delta=0.4 T, \Delta=0.5 T$, where $T$ is an optical cycle of the XUV pulse. Vertical solid line corresponds to the momentum $k_{z}$ for which $E_{0}+\omega=k_{z}^{2} / 2$.

Results of the procedure based on the minimization of the functional (12) are illustrated in Fig. 8, where we present

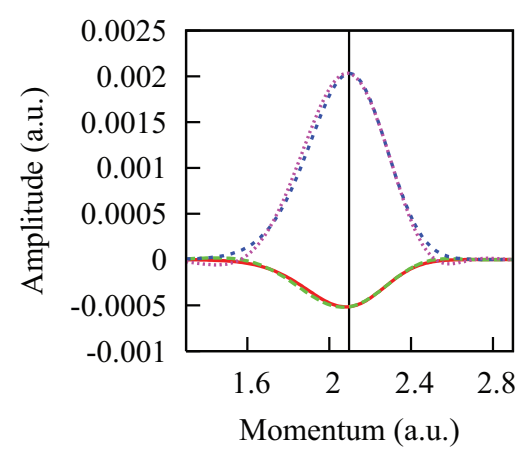

FIG. 6. (Color online) $\operatorname{Re} f^{\mathrm{XUV}}\left(k_{z}, 0\right)$ computed using Eq. (10), solid (red online) line, and TDSE calculation, dashed (green online) line. $\operatorname{Im} f^{\mathrm{XUV}}\left(k_{z}, 0\right)$ given by Eq. (10), short dashed (blue online) line, and TDSE, dotted (magenta online) line. XUV photon energy $\omega=106 \mathrm{eV}$; ionization from the $2 s$ state of Ne. Vertical solid line corresponds to the momentum $k_{z}$ for which $E_{0}+\omega=k_{z}^{2} / 2$.

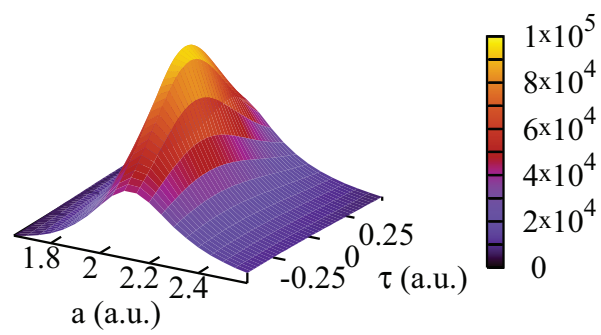

FIG. 7. (Color online) Quantity $D^{-1}\left(a, \tau, \epsilon_{0}\right)$ as function of $a$, and $\tau$. Value of $\epsilon_{0}$ is fixed at the energy conservation value $\epsilon_{0}=E_{0}+\omega$.

the data for the time delay for several base frequencies $\omega$ of the XUV pulse for ionization from the $2 s$ and $2 p$ subshells of the Ne atom. These results are compared with the values for the time delays which we can extract directly from the TDSE calculation using the computed amplitudes of XUV photoionization and Eq. (9).

Figure 8 indicates that the results of the fitting procedure described above agree well with the results of the $a b$ initio TDSE calculation. For completeness, in the same figure we display the time delay results obtained from the Hartree-Fock elastic scattering phases $\tau^{\mathrm{HF}}=d \delta_{l}^{\mathrm{HF}} / d E$. These phases are calculated in the frozen-core Hartree-Fock approximation to electron scattering in the field of the $\mathrm{Ne}^{+}$ion [22]. The scattering phase in the dominant photoionization channel $l=l_{i}+1$ is taken according to the Fano propensity rule [23], where $l_{i}$ is the angular momentum of the initial bound state. Although these results are not directly comparable to the present calculations, which employ a localized effective potential, they demonstrate a qualitatively similar dependence of the time delay on the photon energy. We should emphasize that it is the Hartree-Fock results only that rely explicitly on the Fano propensity rule. In contrast, the time delay defined by Eq. (9) is evaluated using the full amplitude $f^{\mathrm{XUV}}\left(k_{z}, 0\right)$. In the partial wave expansion of the scattering state $\Psi_{k}^{-}$entering this amplitude, both the channels $l=l_{i} \pm 1$ are included.

We may note that the present method of extraction of the attosecond time delay, based on the soft-photon approximation, can be linked to the approach developed in Refs. [10-12],

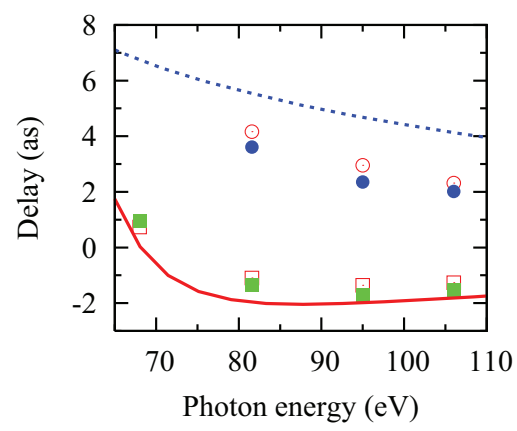

FIG. 8. (Color online) Time delays computed using Eq. (9) and the fitting procedure. Ionization from the $2 s$ state: empty box (red online), Eq. (9); solid box (green online), the fitting procedure. Ionization from the $2 p$ state: empty circle (magenta online), Eq. (9); solid circle (blue online), the fitting procedure. The HF results: $2 s \rightarrow E p$ transition, solid line (red online); $2 p \rightarrow E d$ transition, dashed line (blue online). 
which is a refinement of the strong-field approximation Eq. (1). As we mentioned above, this refinement consists in introducing the correction factor, multiplying the vector potential in Eq. (1), and adding the so-called Coulomb-laser coupling correction in the argument of the function $\boldsymbol{A}^{\mathrm{IR}}(t)$. It is easy to see from Eqs. (8) and (10) that the photoelectron spectrum of the twocolor ionization $P\left(k_{z}, \Delta\right)$ in the soft-photon approximation can be represented as $P\left(k_{z}, \Delta\right)=h\left(k_{z}, \Delta+\tau\right)$, where $\tau$ is the time delay and $h$ is a periodic function of the second argument with the period $T_{1}$ equal to the optical cycle of the IR field. The first moment of $P\left(k_{z}, \Delta\right)$ can therefore be represented as $\bar{k}_{z}(\Delta)=q(\Delta+\tau)$, where $q$ is periodic with period $T_{1}$. We can write, therefore,

$$
\bar{k}_{z}(\Delta)=\sum_{n=0}^{\infty} B_{n} \sin \Omega(\Delta+\tau)+C_{n} \cos \Omega(\Delta+\tau) .
$$

The vector potential of the IR field described by Eq. (2) is $A^{\mathrm{IR}}(t)=\frac{\mathcal{E}_{0}^{\mathrm{IR}}}{\Omega}(1-\cos \Omega t)$. By retaining the terms with $n=0,1$ in Eq. (13) we obtain

$$
\bar{k}_{z}(\Delta)-b \approx c A^{\mathrm{IR}}(\Delta+\tau+\delta)
$$

where the coefficients $b, c$, and $\delta$ can be expressed in terms of the coefficients of the Fourier expansion (13). By identifying the coefficients $b, c$, and $\delta$ in the Eq. (14) with $k_{z}^{0}$, the correction factor multiplying the vector potential in Eq. (1), and the Coulomb-laser coupling correction, we obtain the equation replacing the strong-field relation (1) in the approach developed in Refs. [10-12].

\section{CONCLUSION}

In the present work, we examined applicability of the soft-photon approximation for evaluation of the amplitudes of two-color XUV and IR ionization. We have found that the two-color ionization amplitudes, computed using the soft-photon approximation, agree well with the $a b$ initio TDSE amplitudes. This fact can be used to extract phase information and, in particular, the time delay from the experimental photoelectron spectra detected in attosecond streaking measurements.

We tested the range of validity of the soft-photon approximation. We demonstrated that this approximation renders the two-color ionization amplitudes accurately for the IR field intensities in the range $\mathcal{E}^{\mathrm{IR}}=0.001-0.004$ a.u. (corresponding intensities in the range $3.5 \times 10^{10}$ to $\left.5.6 \times 10^{11} \mathrm{~W} / \mathrm{cm}^{2}\right)$. The softness of the IR photon requires that its frequency should be much less than the kinetic energy of the photoelectron $\Omega / E_{\text {kin }} \ll 1$. This means that the XUV photon energy should be well above the photoionization threshold. This is usually the case in the attosecond time delay measurements to minimize the effect of a large spectral width due to a short XUV pulse. It was found in Ref. [16] that the soft-photon approximation reproduces quite accurately the angle-integrated cross sections for the values of this ratio as large as 0.06 . We observed in the present study that the amplitudes were rendered accurately by the soft-photon approximation for $\Omega / E_{\text {kin }} \approx 0.07$ for the ionization from the inner $2 s$ subshell of the $\mathrm{Ne}$ atom with the
XUV frequency of 2.5 a.u. This defines the lower bound for the XUV frequency, where we can use this approximation safely.

In our numerical examples, we confined ourselves to short XUV and IR pulses, which are used in typical attosecond streaking experiments. However, in deriving our basic Eq. (8), we did not make any assumptions about the pulse duration. We can expect therefore that the applicability of the soft-photon approximation to the two-color ionization process can be extended to longer IR pulses which lead to appearance of side bands in the photoelectron spectrum. It can be used therefore for the timing analysis of the photoelectron spectra obtained in RABITT experiments.

In order to test the soft-photon approximation, we considered photoionization of the neon atom described by a localized one-electron potential. It is a computationally efficient model for which a considerable amount of otherwise time-consuming TDSE calculations could be performed relatively fast. By choosing a deliberately simplified SAE model, we restricted the accuracy of our numerical results, which may not be compared directly with the experimental data. Nevertheless, a fair comparison can be made with other calculations performed in similar SAE approximations [3,12,24]. This comparison can be made for the relative time delay between photoemission from the $2 p$ and $2 s$ subshells of $\mathrm{Ne}$ at the XUV photon energy of $106 \mathrm{eV}$. In the present work, this value is approximately 4 as, both in the soft-photon and TDSE calculations, which is in good agreement with the results reported in Refs. [3,12,24]. The computed value of the relative time delay between photoemission from the $2 p$ and $2 s$ subshells of $\mathrm{Ne}$ is thus not very sensitive to a particular form of the SAE potential used in the calculations. Refinements of the SAE model do not alter this result considerably. With the use of the nonlocal HF potential, relative time delay becomes 6.2 as [24]. With the account of the intershell correlation, this value further increases to 8.4 as [24]. The effect of the intershell correlation in $\mathrm{Ne}$ is thus not as strong as in Ar, where it can revert the sign of the relative time delay between the $3 s$ and $3 p$ subshells [25]. All the figures quoted above are still quite far from the experimental value of $21 \pm 5$ as for the relative time delay between photoemission from the $2 p$ and $2 s$ in $\mathrm{Ne}$ reported in Ref. [3].

In principle, the soft-photon approximation can be used for theoretical studies of two-color ionization in systems described by $a b$ initio Hamiltonians. In Ref. [26], we performed such a study for the process of double ionization of the He atom. The formulas for the amplitudes of the two-color ionization in this case are quite similar in structure to Eq. (8). In order to obtain the ionization amplitude in presence of the IR and XUV fields, one needs to know the "bare" amplitude of the ionization by the XUV field alone as a function of the photoelectron energy. The latter can be furnished by various computational methods. In [26] we obtained the "bare" amplitude by solving the TDSE with the $a b$ initio Hamiltonian of the He atom. We found that the two-color amplitudes, obtained in the soft-photon approximation, and the $a b$ initio ones, provided by the TDSE calculation, agree quite well. In Ref. [26] our concern was to reconstruct the phase of the amplitude from the experimentally observable data. With minor modifications, we can apply this procedure to study time delays for the double ionization in a completely ab initio setting. 


\section{ACKNOWLEDGMENTS}

The authors acknowledge the support of the Australian Research Council in the form of Discovery Grant No.
DP120101805. Facilities of the National Computational Infrastructure National Facility were used.
[1] E. P. Wigner, Phys. Rev. 98, 145 (1955).

[2] C. A. A. de Carvalho and H. M. Nussenzveig, Phys. Rep. 364, 83 (2002).

[3] M. Schultze, M. Fieß, N. Karpowicz, J. Gagnon, M. Korbman, M. Hofstetter, S. Neppl, A. L. Cavalieri, Y. Komninos, T. Mercouris et al., Science 328, 1658 (2010).

[4] P. Eckle, A. N. Pfeiffer, C. Cirelli, A. Staudte, R. Dörner, H. G. Muller, M. Büttiker, and U. Keller, Science 322, 1525 (2008).

[5] P. M. Paul, E. S. Toma, P. Breger, G. Mullot, F. Augé, P. Balcou, H. G. Muller, and P. Agostini, Science 202, 1689 (2001).

[6] J. M. Dahlström, A. L'Huillier, and A. Maquet, J. Phys. B 45, 183001 (2012).

[7] J. M. Dahlström, T. Carette, and E. Lindroth, Phys. Rev. A 86, 061402(R) (2012).

[8] J. M. Dahlström, D. Guénot, K. Klünder, M. Gisselbrecht, J. Mauritsson, A. L'Huillier, A. Maquet, and R. Taïeb, Chem. Phys. 414, 53 (2013).

[9] J. Itatani, F. Quéré, G. L. Yudin, M. Y. Ivanov, F. Krausz, and P. B. Corkum, Phys. Rev. Lett. 88, 173903 (2002).

[10] S. Nagele, R. Pazourek, J. Feist, K. Doblhoff-Dier, C. Lemell, K. Takasi, and J. Burgdörfer, J. Phys. B 44, 081001 (2011).

[11] R. Pazourek, J. Feist, S. Nagele, and J. Burgdörfer, Phys. Rev. Lett. 108, 163001 (2012).
[12] S. Nagele, R. Pazourek, J. Feist, and J. Burgdörfer, Phys. Rev. A 85, 033401 (2012).

[13] M. Ivanov and O. Smirnova, Phys. Rev. Lett. 107, 213605 (2011).

[14] J. Su, H. Ni, A. Becker, and A. Jaroń-Becker, Phys. Rev. A 87, 033420 (2013).

[15] N. M. Kroll and K. M. Watson, Phys. Rev. 8, 804 (1973).

[16] A. Maquet and R. Taïeb, J. Mod. Opt. 54, 1847 (2007).

[17] A. Sarsa, F. J. Gálvez, and E. Buendia, At. Data Nucl. Data Tables 88, 163 (2004)

[18] G. Duchateau, E. Cormier, and R. Gayet, Phys. Rev. A 66, 023412 (2002).

[19] A. S. Kornev and B. A. Zon, J. Phys. B 35, 2451 (2002).

[20] I. A. Ivanov and A. S. Kheifets, Phys. Rev. A 87, 033407 (2013).

[21] I. A. Ivanov, Phys. Rev. A 83, 023421 (2011).

[22] A. S. Kheifets, arXiv:1302.4495.

[23] U. Fano, Phys. Rev. A 32, 617 (1985).

[24] A. S. Kheifets and I. A. Ivanov, Phys. Rev. Lett. 105, 233002 (2010).

[25] D. Guénot, K. Klünder, C. L. Arnold, D. Kroon, J. M. Dahlström, M. Miranda, T. Fordell, M. Gisselbrecht, P. Johnsson, J. Mauritsson et al., Phys. Rev. A 85, 053424 (2012).

[26] I. A. Ivanov and A. S. Kheifets, Phys. Rev. A 83, 063411 (2011). 